\title{
Anti-allergic ophthalmic drugs in general practice: which, why and when?
}

\author{
Mohamed N, MBChB, FC Ophth(SA), Registrar; Smit DP, MBChB, MMed(Ophth), FCOphth(SA), Consultant \\ Division of Ophthalmology, Faculty of Health Sciences, Stellenbosch University, and Tygerberg Hospital \\ Correspondence to: Derrick Smit, e-mail: dpsmit@sun.ac.za \\ Keywords: anti-allergic ophthalmic drugs, general practice
}

\begin{abstract}
Ocular allergies present in many different guises. They may vary from self-limiting episodes of acute allergic conjunctivitis, to potentially sight-threatening chronic conditions, such as vernal keratoconjunctivitis. This article provides a brief description of the different ocular allergic conditions to aid accurate diagnosis. It also focuses on the different types of anti-allergic ophthalmic preparations and how to employ them in a logical, stepwise management strategy to obtain optimal results, while minimising exposure to potent drugs with potentially serious side-effects.
\end{abstract}

(P) Peer reviewed. (Submitted: 2012-11-13. Accepted: 2013-02-07.) ๑ SAAFP

S Afr Fam Pract 2013;55(4):313-318

\section{Introduction}

Allergic or immune-mediated reactions of the conjunctiva are not uncommon. This may, in part, be because the conjunctiva has a rich vascular supply, abundant immune mediators and direct exposure to the environment. ${ }^{1}$ Allergic conjunctivitis encompasses a wide spectrum, from acute, self-limiting forms of seasonal conjunctivitis, to more chronic, sight-threatening forms of vernal and atopic keratoconjunctivitis.

The major forms of allergic conjunctivitis involve a type 1 hypersensitivity reaction, mediated by the degranulation of mast cells in response to immunoglobulin $E$ in genetically susceptible individuals upon exposure to certain environmental antigens to which they have previously been exposed. There is also evidence of type IV hypersensitivity in at least some forms. ${ }^{2,3}$

The purpose of this article was to review the different antiallergic ophthalmic drugs in the same sequence in which they should be considered in the treatment of a patient with an ocular allergy. Thereafter, short descriptions of ocular allergic conditions follow to aid accurate diagnosis and initiation of appropriate stepwise therapy to reduce the potentially adverse effects of allergic conjunctivitis.

\section{An approach to the treatment of allergic ocular conditions}

A variety of agents is currently available to treat allergic conjunctivitis. These are used either alone or in combination with each other (Table I):
- Elimination or avoidance of the specific allergen: Elimination or avoidance of the specific allergen is a crucial first step. Although this is not always possible or practical, allergy specialists can help with the identification and avoidance of the offending allergen prior to recommending any allergy remediation measures.

- Lubricants: Chilled, unpreserved lubricants, such as polyvinyl alcohol or povidone, e.g. Refresh ${ }^{\circledR}$, or hypromellose or dextran, e.g. Tears Naturale ${ }^{\circledR}$, help dilute and flush antigens from the ocular surface, and thus aid in providing symptomatic relief. ${ }^{4}$

- Cold compresses: Cold compresses, consisting of ice packs wrapped in a clean cloth, may be applied over the closed eyes to provide symptomatic relief. ${ }^{2}$

- Topical vasoconstrictors: Topical vasoconstrictors, such as oxymetazoline and nephazoline, cause vasoconstriction and decrease vascular permeability. They may be used alone, eg. Allergex ${ }^{\circledR}$, or in combination with antihistamines such as antazoline, e.g. Spersallerg ${ }^{\circledR}$. These provide short-term symptomatic relief, but should not be used for longer than two weeks because of rebound hyperaemia.

- Topical and oral antihistamines: Topical and oral antihistamines act by competitive inhibition of histamine at the histamine- 1 and histamine- 2 receptors, and are effective in relieving the acute signs and symptoms of allergic eye conditions. Systemic antihistamines are indicated for severe symptoms, but may be associated with adverse effects, such as drowsiness, particularly in the case of the older antihistamines. Newer oral antihistamines, such as cetirizine, e.g. Zyrtec $^{\circledR}$, and desloratadine, e.g. Deselex ${ }^{\circledR}$, have fewer 
side-effects, and are therefore more preferable. Topical antihistamines, such as levocabastine, e.g. Livostin $\mathrm{ED}^{\circledR}$, and emedastine, e.g. Emadine ${ }^{\circledR}$, have a more rapid onset and are less likely to cause systemic side-effects, when compared to oral preparations. However, their actions are generally short-lived, and thus they are preferred in milder cases.

- Topical mast cell stabilisers: Topical mast cell stablisers inhibit the degranulation of mast cells, thus limiting the release of inflammatory mediators, such as histamine. Available agents include sodium chromoglycate, e.g. Chromohexal $^{\circledR}$, and lodoxamide, e.g. Alomide ${ }^{\circledR}$. These agents play a largely preventative role and need to be used for a few weeks before exerting their maximal effect. ${ }^{2}$ It is important to bring this fact to the attention of the user.

- Agents with multiple mechanisms of action (MMA): Agents with MMA, such as olopatidine, e.g. Patanol ${ }^{\circledR}$, and ketotifen, e.g. Zaditen ${ }^{\circledR}$, have both antihistamine and mast cell-stabilising properties, and are often the preferred choice for ophthalmologists and their patients.

- Topical nonsteroidal anti-inflammatory drugs (NSAIDs): Topical NSAIDs, such as ketorolac, e.g. Acular ${ }^{\circledR}$, and diclofenac, e.g. Voltaren Ophtha ${ }^{\circledR}$, inhibit the activity of cyclo-oxygenase and may provide added benefit in reducing the signs and symptoms associated with allergic conjunctivitis. ${ }^{1,2}$

- Topical corticosteroids: Topical corticosteroids inhibit the inflammatory process and are effective in reducing the acute symptoms of ocular allergy. Usually, they are prescribed in short, intensive courses and promptly tapered thereafter. ${ }^{2}$ Potent preparations, such as prednisolone $1 \%$, are generally effective, but their protracted use has been associated with wellknown complications, such as glaucoma and cataract formation. Newer, less potent corticosteroids, such as fluorometholone $0.1 \%$, loteprednol $0.2-0.5 \%$ and rimexolone $1 \%$, have been designed to lower the risk of these side-effects, but are generally not as effective as
Table I: Commonly used topical agents in the treatment of allergic conjunctivitis

\begin{tabular}{l|l|l|}
\hline Agent & Concentration (\%) & $\begin{array}{l}\text { Frequency of } \\
\text { administration }\end{array}$ \\
\hline $\begin{array}{l}\text { Antihistamines } \\
\text { Levocabastine }\end{array}$ & 0.05 & q.i.d. \\
\hline $\begin{array}{l}\text { Mast cell stabilisers } \\
\text { Sodium } \\
\text { chromoglycate }\end{array}$ & 4 & q.i.d. \\
\hline Lodoxamide & 0.1 & q.i.d. \\
\hline Antihistamines and mast cell stabilisers & \\
\hline $\begin{array}{l}\text { Olopatadine } \\
\text { Nonsteroidal anti-inflammatory drugs }\end{array}$ & b.i.d. \\
\hline Ketolorac & 0.5 & q.i.d. \\
\hline Corticosteroids & & \\
\hline Dexamethasone & 0.1 & Variable \\
\hline Fluorometholone & 0.1 & Variable \\
\hline $\begin{array}{l}\text { Prednisolone } \\
\text { acetate }\end{array}$ & $0.12-1$ & Variable \\
\hline Immunomodulatory & $0.05-2$ & \\
\hline Cyclosporine & 0.03 & Variable \\
\hline Tacrolimus & & \\
\hline b.i.d. & \\
\hline
\end{tabular}

b.i.d: twice a day, q.i.d: four times a day

their more potent counterparts. Steroid injections above the tarsal plate in the eyelid may also be given in severe palpebral disease or to patients who are refractory to initial therapy. ${ }^{2}$

- Topical T-cell inhibitors: Topical T-cell inhibitors, such as cyclosporine $1 \%$ and tacrolimus $0.03 \%$, e.g. Protopic ${ }^{\circledR}$, may be indicated as steroid-sparing agents, or in severe refractory disease under specialist supervision. Cyclosporine has been associated with ocular irritation and relapses when stopped abruptly. ${ }^{2}$ Tacrolimus ointment has been shown to be very effective in modulating conjunctival inflammation in refractory cases of ocular allergy. ${ }^{2,5}$

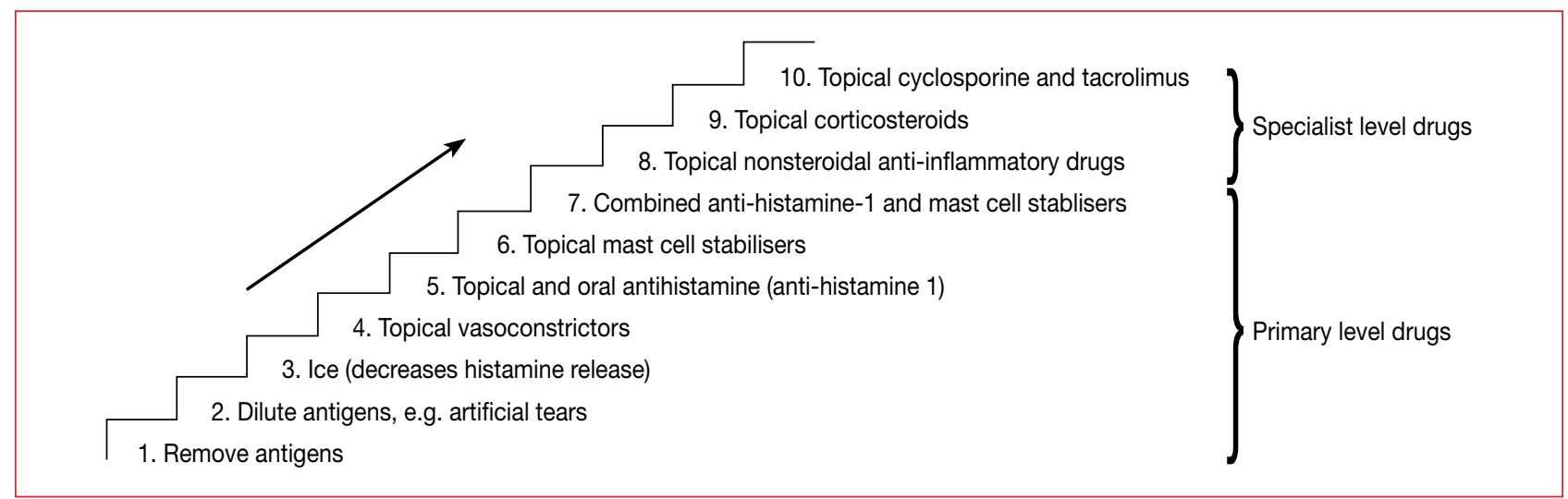

Source: Prof D Meyer, Division of Ophthalmology, Faculty of Health Sciences, Stellenbosch University

Figure 1: Stepwise approach to the management of allergic conjunctivitis 


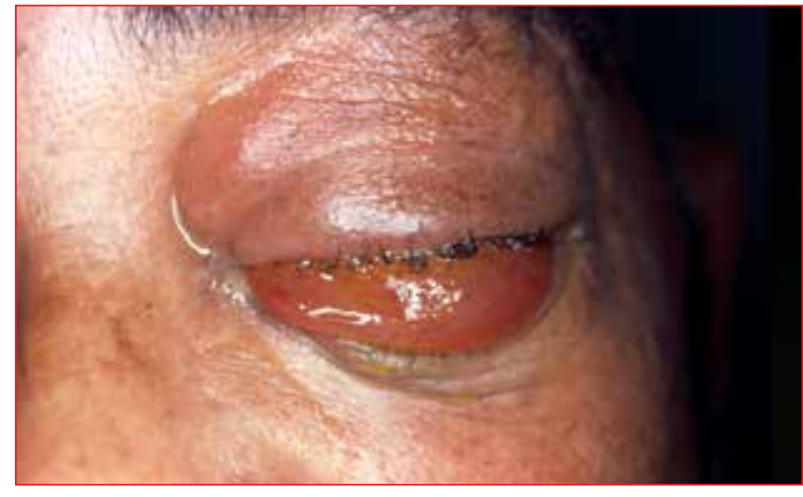

Figure 2: Acute allergic conjunctivitis: Conjunctival chemosis and lid swelling

A stepwise approach to the management of ocular allergies is important in preventing unnecessary or inadvertent overtreatment of the underlying condition (Figure 1).

\section{Acute allergic conjunctivitis}

Acute allergic conjunctivitis describes the immediate response seen in sensitised individuals, typically children, after exposure to a particular allergen or sensitising agent, usually pollen or cat dander. ${ }^{2}$

\section{Clinical picture and management}

Acute allergic conjunctivitis is characterised by episodes of intense itching and watering, associated with severe conjunctival chemosis and lid swelling (Figure 2).

The condition is self-limiting and settles promptly with the removal of the offending allergen. Cool compresses and artificial tears can be applied to reduce symptoms considerably, but topical and/or oral antihistamines may be required in selected cases, i.e. management steps 1-5.

\section{Seasonal and perennial allergic conjunctivitis}

Seasonal allergic conjunctivitis, also known as hay fever conjunctivitis, represents the most common form of allergic conjunctivitis. ${ }^{6}$ The seasonal nature of the condition relates to the appearance of specific allergens, most notably tree and grass pollens, although the specific allergen may vary with geographical location. There is often associated rhinitis and/or a history of atopy.

Perennial allergic conjunctivitis represents a more chronic form. Generally, signs and symptoms are present throughout the year. Notable allergens include house dust mites, animal dander and mould.

\section{Clinical picture and management}

Patients present with transient attacks of ocular itching, tearing and redness. Classic examination findings include conjunctival injection (Figure 3), eyelid oedema, as well as varying degrees of chemosis (conjunctival oedema).

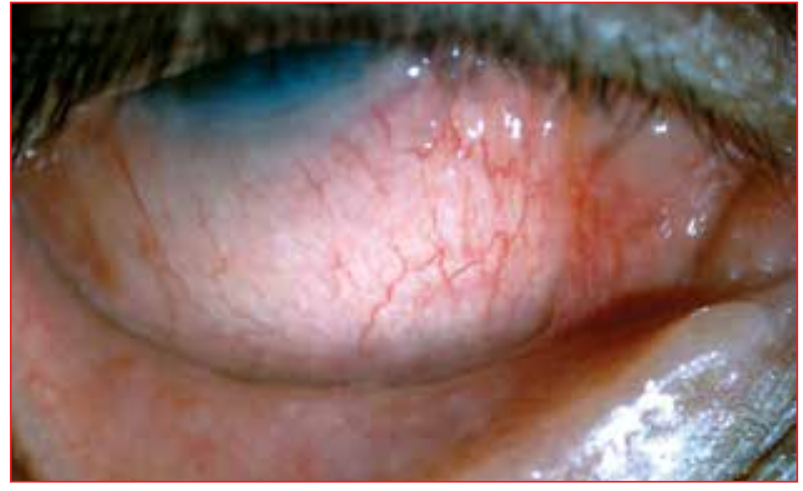

Figure 3: Seasonal and perennial allergic conjunctivitis: Acute conjunctival injection

Management starts with the identification and removal of the offending allergen, if possible. Mild cases should respond to antigen dilution, cold compresses and shortterm use of topical vasoconstrictors, i.e. management steps $2-4$. Moderate cases may require the addition of topical and oral antihistamines, i.e. management step 5, while more severe and/or chronic cases often require the use of topical mast cell stabilisers, MMAs and topical corticosteroids, i.e. management steps 6-9.

\section{Vernal and atopic keratoconjunctivitis}

Vernal keratoconjunctivitis (VKC) is a chronic bilateral corneal and conjunctival inflammatory condition that predominantly affects young boys who often have a personal or family history of atopy. The disease is more common in warm, dry climates, where patients may experience symptoms throughout the year. In temperate climates, VKC tends to be seasonal and often flares up during spring. Fortunately, the frequency and severity of attacks subsides in most patients as they reach puberty.

\section{Clinical picture and management}

VKC characteristically presents with symptoms of intense itching, associated with tearing, photophobia, a mucoid discharge and a foreign body sensation. The disease usually presents in two ways, either as a palpebral or limbal type, although both may coexist in a patient.

In the palpebral form, there is diffuse papillary hypertrophy under the upper lid that is associated with thick mucoid deposition between the papillae (Figure 4). The limbal form is characterised by the presence of gelatinous papillae on the limbal conjunctiva, which often contain distinct white nodules called Horner-Trantas dots (Figure 5). This form is frequently seen in more pigmented patients who live in hotter climates. The cornea may also be affected with erosions and dry white plaques called shield ulcers in severe cases (Figure 6).

Similar to VKC, atopic keratoconjunctivitis (AKC) is a severe, chronic form of ocular allergy which presents 


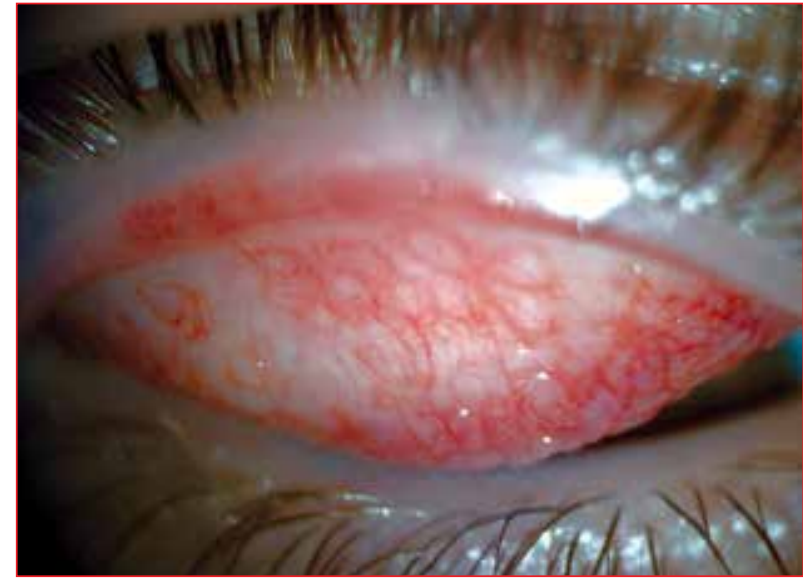

Figure 4: Palpebral vernal disease: Diffuse subtarsal papillae

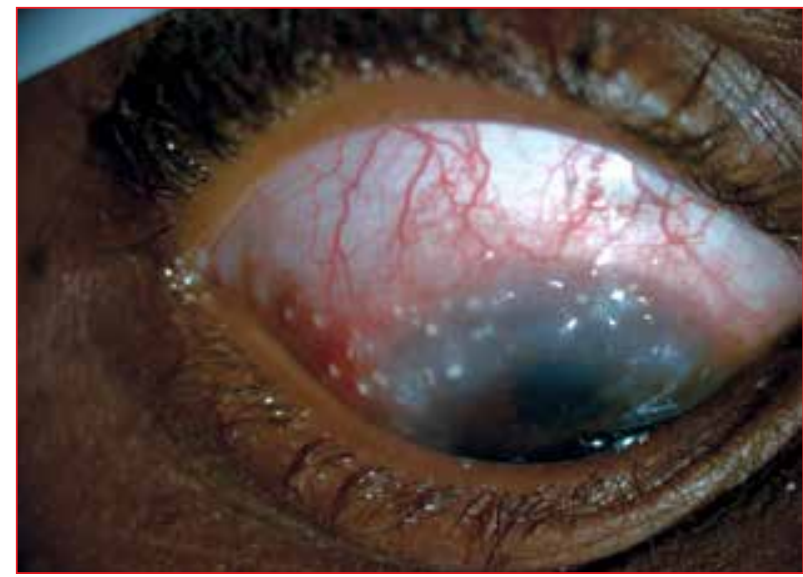

Figure 5: Limbal vernal disease: Limbal papillae and Horner-Trantas dots

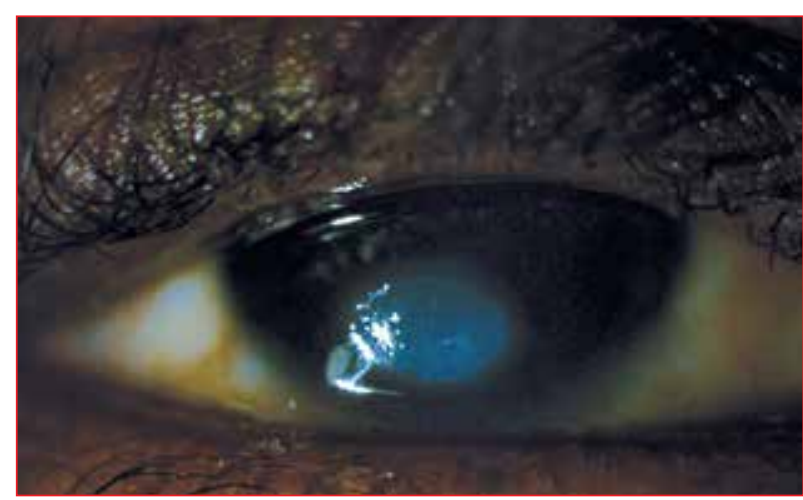

Figure 6: Corneal involvement in vernal disease: Shield ulcer and plaque

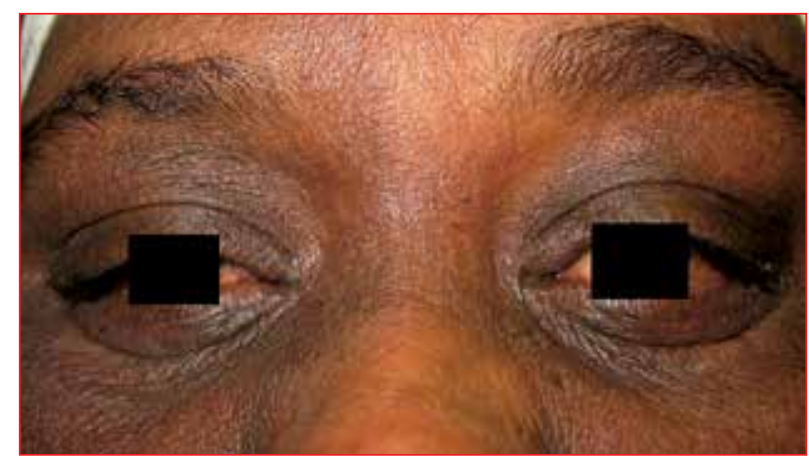

Figure 7: Eyelid thickening and keratinisation: Eyelid thickening and keratinisation in atopic keratoconjunctivitis with symptoms of intense itching, mucoid discharge, photophobia and blurry vision. It has a strong association with atopic dermatitis. Features that distinguish AKC from VKC are the location of papillae under both the upper and lower eyelids, as opposed to only the upper eyelid in VKC. Perennial symptoms and onset, beginning in the second or third decade, are other distinguishing features. Eyelid changes, such as thickening and keratinisation of eyelid margins, are also more prevalent in AKC (Figure 7).

The management of VKC is similar to that of AKC, and involves a multipronged approach with environmental, systemic and topical measures to control the disease. Treatment duration is typically long term and requires frequent follow-up. Allergen avoidance is important in the treatment process, i.e. management step 1 , similar to that in other atopic conditions. While antigen dilution, cold compresses, topical vasoconstrictors and antihistamines provide short-term relief, topical mast cell stabilisers may reduce both the frequency of acute exacerbations and the need for corticosteroids. Therefore, they form the cornerstone of treatment, i.e. management steps 2-6. Topical MMAs and NSAIDs are useful treatment options in moderate cases, i.e. management steps 7 and 8 . Topical immunomodulatory drugs, such as corticosteroids and cyclosporine, are often required in severe or refractory cases, but should preferably be taken under specialist supervision, i.e. management steps 9 and 10.

\section{Giant papillary conjunctivitis}

Giant papillary conjunctivitis (GPC) is typically seen in association with contact lens wear. However, it may also be associated with a variety of other foreign objects, such as ocular prostheses or exposed sutures.

\section{Clinical picture and management}

Symptoms of GPC include a foreign body sensation, itching, redness and decreased contact lens tolerance. A mucoid discharge is also commonly present. Eversion of the upper lid reveals giant papillae, similar to those seen in VKC. The papillae are easily visible, but examination with a cobalt blue light, after installation of topical fluorescein, delineates the papillae more clearly, and in severe cases, apical staining of the papillae may be observed. ${ }^{4}$

Management may require the use of almost all of the steps, but begins, as always, with the removal of any offending stimuli. In cases that are triggered by contact lens wear, reducing the wearing time, increasing the frequency of lens replacement, improving lens cleaning to reduce protein build-up, as well as changing the lens design or material, may prove to be helpful.

Topical antihistamines, mast cell stabilisers and combination agents can all provide relief from the symptoms. Usually, 
topical steroids are reserved for use in severe, refractory cases of GPC. Ideally, these patients should be managed in conjunction with an ophthalmologist.

\section{Toxic conjunctivitis}

Toxic conjunctivitis, while not truly allergic in nature, is frequently confused with allergic conjunctivitis, and may occur following the administration of ophthalmic medication. ${ }^{7}$ Preservatives in ophthalmic medication and contact lens solution, most notably benzalkonium chloride and thiomersal, are the most common causes.

\section{Clinical picture and management}

Unilateral or bilateral conjunctival hyperaemia and a mixed follicular or papillary reaction of the palpebral conjunctiva are characteristic initial findings. Ideally, this is seen with the aid of a slit lamp, but may be observed with a direct ophthalmoscope, if large enough. Occasionally, progressive conjunctival scarring may ensue and lead to contraction of the conjunctival fornices. Corneal punctate epithelial erosions, especially of the inferior cornea, may also be present.

Treatment involves identifying and removing the offending agent. Symptomatic patients may benefit from using cold compresses and preservative-free artificial tears, i.e. management steps 1-3.

\section{Conclusion}

Allergic conjunctivitis represents a diverse group of disorders, with many overlapping clinical manifestations. Distinguishing between the various subtypes is important and can be achieved by taking a thorough history and conducting a careful clinical observation. Avoidance of the offending allergen is the primary behavioural modification for all types of allergic conjunctivitis, and serves as the starting point for a common stepwise approach to the management of ocular allergy.

\section{References}

1. Care of the patient with conjunctivitis. American Optometric Association [homepage on the Internet]. 2002. c2012. Available from: http://www.aoa.org/ documents/CPG-11.pdf

2. Kanski JJ. Clinical ophthalmology: a systematic approach. $6^{\text {th }}$ ed. Edinburgh: Elsevier ButterworthHeinemann; 2007

3. Rothenburg ME, Owen WF Jr, Stevens RL. Ocular allergy. Mast cells and eosinophils. Int Ophthalmol Clin. 1988; 28(4):267-274

4. Rossiter D, Blockman M, Barnes KL, et al, editors. South African medicines formulary. $10^{\text {th }}$ ed. Cape Town: Health and Medical Publishing Group; 2012.

5. Garcia DP, Alperte JI, Cristóbal JA, et al. Topical tacrolimus ointment for treatment of intractable atopic keratoconjunctivitis: a case report and review of the literature. Cornea. 2011;30(4):462-465.

6. Gupta V, Tandon R, Vajpayee RB. Disorders of the conjunctiva. Textbook of ophthalmology. $5^{\text {th }}$ ed. New Delhi: Jaypee Brothers, 2002; p. 843-849.

7. Nema HV, Nema N. Diseases of the conjunctiva. Textbook of ophthalmology. $5^{\text {th }}$ ed. New Delhi: Jaypee Brothers, 2002; p. 109-141.

\section{Sandoz launches Nexmezol, treatment for digestive disorders, in South Africa}

Sandoz, the generic pharmaceuticals division of Novartis, has launched Nexmezol. Nexmezol is the first generic esomeprazole in South Africa.

Nexmezol is indicated for the treatment of gastro-oesophageal reflux disease (GORD), a digestive disorder that affects the lower oesophageal sphincter, the muscle connecting the oesophagus with the stomach.

In South Africa alone, more than 4,2 million boxes of prescription medicine for heartburn are sold annually ${ }^{1}$. Poor diet and a stressful lifestyle are contributing factors.

"Lifestyle diseases, coupled with high costs make it increasingly difficult for South African patients to gain access to affordable treatment for their healthcare problems. Medical aids often do not pay for medicines that treat gastro-oesophageal reflux disease, therefore it is mostly funded out of the acute/savings portion of medical aids " says Carnie van der Linde Country Head for South East Africa.

Research shows that patients with GORD are bothered and even incapacitated by their symptoms, and that their health-related quality of life is impaired as a result. ${ }^{2}$ Patients with heartburn experience worse pain, social functioning and emotional wellbeing than those with diabetes, hypertension and acute coronary events. ${ }^{3}$
The proton pump inhibitor (PPI) in Nexmezol is often a solution for sufferers who have tried other alternatives. Nexmezol is effective in acid suppression. ${ }^{4}$

\section{References}

1. IMS TPM May 2013 - Schedule 3 and 4

2. The South African Gastroenterology Review, November 2005: What is the burden of illness in patients with reflux disease in South Africa? CJ Van Rensburg, KR Kulich, J Carlsson and IK Wiklund.

3. Quality of life in acute and maintenance of treatment of non-erosive and mild erosive gastro-oesophagel reflux disease. F. Pace, C. Negrini, I Wiklund, C. Rossi and V. Savarino.

4. Approved package insert.

\section{For more information:}

Kholiswa Lekalake, Sandoz South Africa, +2711929 9021

Kholiswa.lekalake@sandoz.com

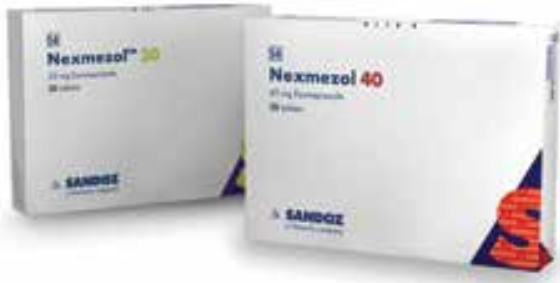

